\title{
THE
}

1994

\section{Locating Transition States Using Double-Ended Classical Trajectories}

\author{
A. Matro \\ University of Rhode Island \\ David L. Freeman \\ University of Rhode Island, dfreeman@uri.edu \\ Jimmie D. Doll
}

Follow this and additional works at: https://digitalcommons.uri.edu/chm_facpubs

Terms of Use

All rights reserved under copyright.

\section{Citation/Publisher Attribution}

Matro, A., Freeman, D. L., \& Doll, J. D. (1994). Locating transition states using double-ended classical trajectories. Journal of Chemical Physics, 101(12), 10458-10463. doi: 10.1063/1.467863

Available at: http://dx.doi.org/10.1063/1.467863 


\title{
Locating transition states using double-ended classical trajectories
}

\author{
A. Matro and D. L. Freeman \\ Department of Chemistry, University of Rhode Island, Kingston, Rhode Island 02881 \\ J. D. Doll \\ Department of Chemistry, Brown University, Providence, Rhode Island 02912
}

(Received 6 September 1994; accepted 15 September 1994)

\begin{abstract}
In this paper we present a method for locating transition states and higher-order saddles on potential energy surfaces using double-ended classical trajectories. We then apply this method to 7 - and 8-atom Lennard-Jones clusters, finding one previously unreported transition state for the 7-atom cluster and two for the 8-atom cluster. (C) 1994 American Institute of Physics.
\end{abstract}

\section{INTRODUCTION}

Classical dynamics calculations in multidimensional systems are most commonly carried out by numerically propagating Newton's equations of motion. This approach requires that initial momenta of the particles be specified as well as initial coordinates. Although this approach has been invaluable in numerous applications such as Molecular Dynamics simulations, there are certain problems in which the use of double-ended boundary conditions can be a more convenient choice.

In the double-ended formulation of the dynamics, the initial and the final coordinates of the system are specified rather than the initial coordinates and momenta. Trajectories that connect the initial and the final configurations of the system are then determined. Doll, Beck, and Freeman ${ }^{1}$ proposed an approach to obtain double-ended trajectories by using the Fourier expansion about the linear constantvelocity path connecting the two configurations. Doubleended classical trajectories were recently constructed by Cho, Doll, and Freeman ${ }^{2}$ to investigate the dynamics of the Henon-Heiles potential. Both bound and dissociative trajectories were located for this potential. In other recent work, Gillian and Wilson have used a discretized formulation of double-ended trajectories to study the dynamics of isomerization and reactive collisions. ${ }^{3}$ In related work, Pratt has outlined a method based on Monte Carlo sampling for locating transition states using the reactant and product configurations as the boundary conditions in the construction of reactive trajectories. ${ }^{4}$

Our goal in this paper is to use the double-ended trajectory approach to find transition states connecting a pair of potential energy minima. The use of double-ended trajectories seems natural in the search for transition-state geometries since an initial and a final configuration of the system are specified explicitly. Although there might exist many trajectories that connect a pair of potential energy minima in a given transit time, it is possible to identify those trajectories that take the system through or close to an extremum on the potential energy surface. In this paper we will refer to trajectories that pass through a transition state as transition-state trajectories. Furthermore, it is possible to restrict the search procedure to favor the low-energy trajectories that are likely to be transition-state trajectories.

Other methods previously employed in transition state searches include the method developed by Cerjan and Miller. ${ }^{5}$ This method uses a procedure that maximizes the potential energy of the system with respect to displacement of one normal mode while simultaneously minimizing the potential energy with respect to displacements of the remaining normal modes. The system, therefore, follows a particular normal mode until a transition state is reached. In recent work, Davis et al. ${ }^{6}$ and Wales ${ }^{7}$ have employed this method as well as the method of slowest slides ${ }^{8}$ in locating transition states in a wide class of systems including atomic LennardJones and ionic clusters.

This paper is organized as follows. Section II reviews the formalism behind the construction of double-ended classical trajectories and describes the criteria for identifying transition-state trajectories. Because the transition-state trajectory for a given transit time must be the lowest energy trajectory connecting a pair of potential energy minima, the computational procedure is tailored to locate simple lowenergy trajectories. In Sec. III, we use this method to locate transition states between isomers of atomic Lennard-Jones clusters. For the 7- and 8-atom clusters we are able to locate previously unreported low-lying transition state(s). Concluding remarks are given in Sec. IV.

\section{METHOD}

The expressions in this section are presented for a onedimensional system to keep the notation simple. Extension to three dimensions and many particles is straightforward. The path traversed by a particle between points $x_{1}$ and $x_{2}$ in time $t$ (referred to from now on as transit time) can be written using a Fourier sine expansion about the constant-velocity path, ${ }^{1,2}$

$$
x(u)=x_{1}+\left(x_{2}-x_{1}\right) u+\sum_{k=1}^{\infty} a_{k} \sin (k \pi u),
$$

where $u \equiv \tau / t$ is the dimensionless time ranging from 0 to 1 , $\left\{a_{k}\right\}$ is the set of expansion coefficients that describes the deviation of the trajectory from a constant-velocity path, and $\tau$ is the physical time along the trajectory. The expansion coefficients $\left\{a_{k}\right\}$ may be obtained by substituting Eq. (1) into Newton's equations of motion or equivalently by requiring 
that the action be stationary with respect to variations of the expansion coefficients. The resulting coefficients are given by ${ }^{1,2}$

$$
a_{k}=-\frac{t^{2}}{m \pi^{2} k^{2}} f_{k}(\mathbf{a}),
$$

where $m$ is the mass of the particle and $f_{k}(\mathbf{a})$ is the $k$ th Fourier sine component of the force on the particle, given by

$$
f_{k}(\mathbf{a})=2 \int_{0}^{1} d u \sin (k \pi u)\left(-\frac{d V(x(u))}{d x(u)}\right) .
$$

The boldface $\mathbf{a}$ in Eqs. (2) and (3) implies that each component $f_{k}($ a) generally depends on the entire set of expansion coefficients. The coefficients $a_{k}$ can be determined by finding the zeros of the function

$$
\chi(\mathbf{a})=\sum_{k=1} \delta_{k}^{2}\left[a_{k}+\frac{t^{2}}{m \pi^{2} k^{2}} f_{k}(\mathbf{a})\right]^{2},
$$

where $\delta_{k}$ are arbitrary real constants. In a previous application $^{2}$ it was convenient to introduce the weighting factors, $\delta_{k}$, to move between regions of $\chi(\mathbf{a})$ that correspond to different paths. In the current work, we have set $\delta_{k}=1$. Each zero of the function $\chi(\mathbf{a})$ corresponds to a classical path that can be traversed by the system between $x_{1}$ and $x_{2}$ in the specified transit time.

The strategy used by Cho, Doll, and Freeman in locating classical trajectories on a Henon-Heiles potential consisted of randomly choosing values for the finite set of expansion coefficients and then minimizing $\chi(\mathbf{a})$ given in Eq. (4). ${ }^{2}$ Their goal was to find all possible trajectories for a given transit time. As the transit time was increased, larger numbers of coefficients were required in the Fourier expansion of the trajectory to describe the increasingly complicated motions undertaken by the particle.

It is not our goal in this work to determine all possible trajectories that take the system from one configuration to another. Instead, we wish to restrict our search to yield only simple low-energy trajectories that can take the system through or close to an extremum on the potential energy surface. The transition states of atomic Lennard-Jones clusters have been recently studied in detail ${ }^{6.7}$ and we choose these systems to test our method. The Lennard-Jones potential is given by

$$
V=4 \epsilon \sum_{i<j}\left[\left(\frac{\sigma}{r_{i j}}\right)^{12}-\left(\frac{\sigma}{r_{i j}}\right)^{6}\right],
$$

where $\epsilon$ is the parameter governing strength of the interaction, $\sigma$ determines the pair equilibrium separation, and $r_{i j}$ is the distance between atoms $i$ and $j$.

The strategy that we employ here consists of using a small number of expansion coefficients for each degree of freedom to exclude the possibility of complicated trajectories from our search. We are looking for the most direct trajectory between the two points on the potential energy surface. The family of trajectories on which we focus is analogous to the trajectories giving rise to the left-most branch in the energytime diagram for dissociation on the Henon-Heiles potential in Fig. 2 of Ref. 2. In addition, transitions from one local minimum to another often involve motion of only a few atoms in the cluster, because most rearrangements in small systems can be described with either of two mechanisms: the Johnson edge-bridging mechanism ${ }^{9}$ or the Lipscomb diamond-square-diamond mechanism. ${ }^{10}$ of course the kinds of rearrangements in large many-body systems can be complex and collective, requiring an analysis beyond the scope of the current work. The simplification described above allows us to further restrict our search by randomly choosing the expansion coefficients for the coordinates of only those atoms that move significantly during the rearrangement, while the rest of the expansion coefficients are initially set to zero. All the coefficients are, of course, optimized in the search for the zeros of $\chi(\mathbf{a})$. The minimization of $\chi(\mathbf{a})$ is performed using the conjugate gradient method. ${ }^{11}$ For all the transition state searches carried out in this work, three expansion coefficients for each Cartesian coordinate are used and it is only necessary to randomly choose the $k=1$ coefficients for the atoms whose positions change significantly during the rearrangement. The range used to generate the initial values
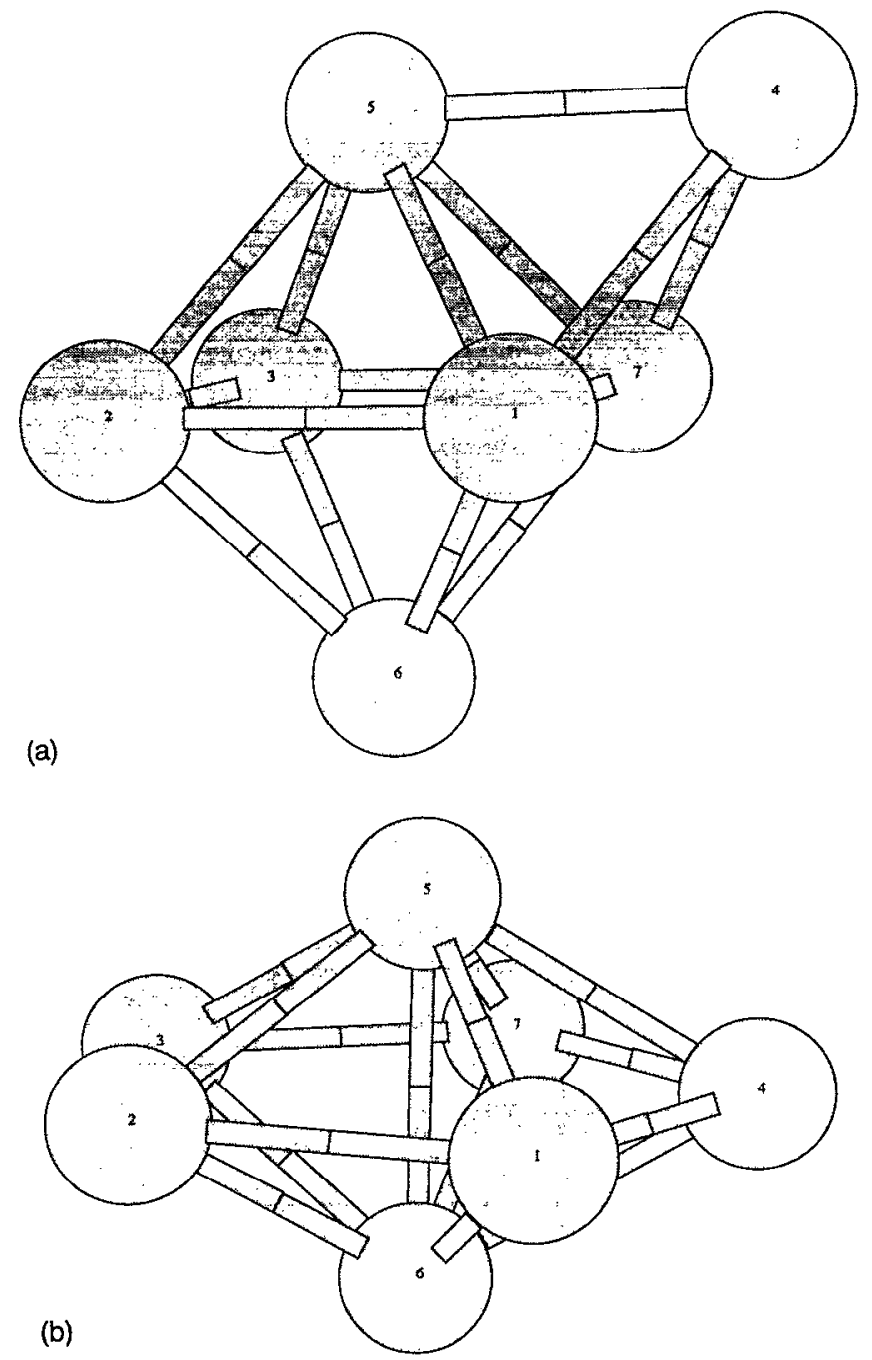

FIG. 1. Local minima of the 7-atom Lennard-Jones cluster: (a) capped octahedron $-15.94 \epsilon$, (b) pentagonal bipyramid $-16.51 \epsilon$, 
of the randomly chosen expansion coefficients is varied from $\pm 0.1 \sigma$ to $\pm 1.0 \sigma$ depending on the particular pair of potential energy minima.

In order for the search to be most efficient, the relative orientations for each pair of minima must be carefully chosen so that as few atoms as possible move during the rearrangement. Figure 1 shows the relative orientation of a pair of potential energy minima of the 7-atom cluster that yields the transition-state trajectory efficiently. Note that the only atom that moves significantly is atom 4. Proper numbering of the atoms in the two clusters is important as well, since inconsistent numbering of atoms in the two structures will force a complicated rearrangement.

A parameter that must be chosen with some care is the transit time. The transit time cannot be too short, since that would result in large kinetic energies, leading to trajectories that are not very sensitive to the underlying potential energy surface (see the short-time behavior of the energy-time diagram in Fig. 2 of Ref. 2). If the transit time is chosen to be too short, the kinetic energy required to complete the trajectory will greatly exceed the potential energy of the cluster, resulting in ballistic trajectories. On the other hand, if a transit time is chosen to be too long, then there is more opportunity to find an undesirable (i.e., complicated) trajectory. We have found that using a transit time that is about 8-10 times longer than the vibrational period of a Lennard-Jones dimer to be most optimal in determining the transition-state trajectories for Lennard-Jones clusters.

Finally, we need to distinguish between those trajectories that pass through or close to the transition state and those that do not. A transition-state trajectory will have the following properties: (a) the total energy of a transition-state trajec-

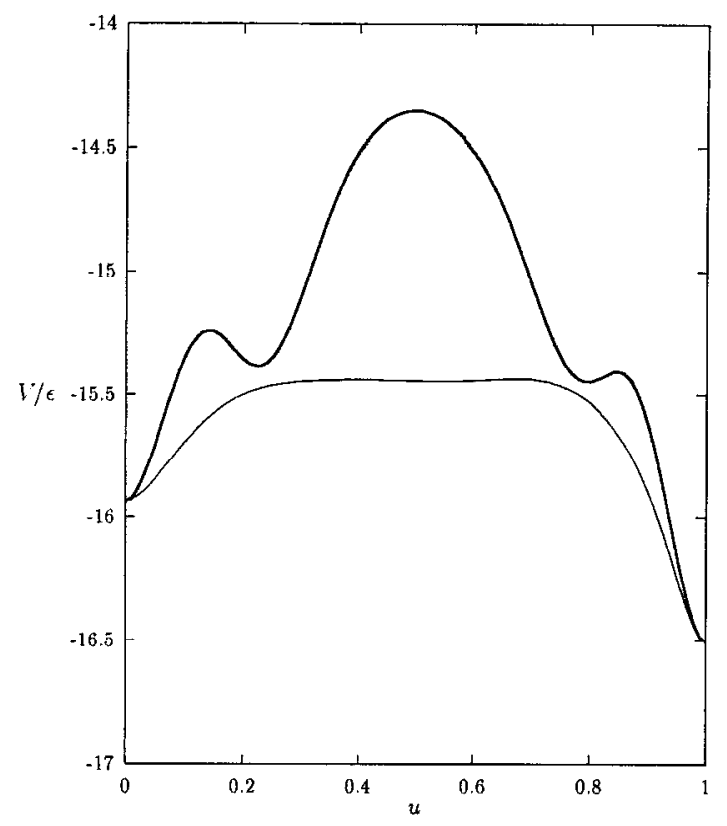

FIG. 2. Potential energy of 7-atom cluster along the trajectory connecting structures shown in Figs. I(a) and I(b). Lower (thin) curve corresponds to a transition-state trajectory, and upper (thick) curve is for a higher-energy trajectory.

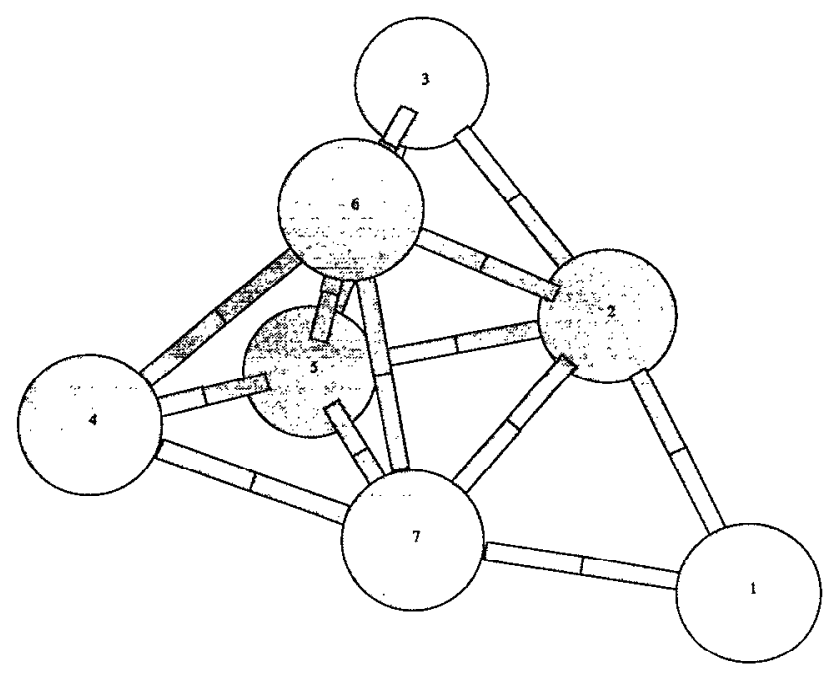

FIG. 3. Transition state between two tricapped octahedra, $V=-14.57 \epsilon$.

tory must be the lowest of all the trajectories found between a particular pair of local minima; and (b) the kinetic energy of the system will just be barely enough to allow the system to go over the barrier. From these two properties we see that the potential energy plotted as a function of time will be nearly constant for a significant portion of transit time as the system slowly moves through the transition state. Figure 2 shows the plot of the potential energy as a function of the dimensionless time $u$ for a transition-state trajectory (thin curve) and a non-transition-state trajectory (thick curve) between the two minima shown in Fig. 1. Both curves begin and end at the potential energy corresponding to the structures shown in Figs. 1(a) and 1(b), respectively. The curve corresponding to the transition-state trajectory is dominated by a prominent plateaulike region throughout a large part of the trajectory. The large zero-slope portion of this curve suggests that the cluster spends a significant portion of its trajectory in a region of the potential energy surface where the forces vanish, i.e. near an extremum. If the cluster is given barely enough kinetic energy to surmount the barrier, it will move extremely slowly near the extremum. As the transit time is increased, the size of the plateaulike region of the time-dependent potential energy curve increases as well. The thick curve in Fig. 2 exhibits three maxima and no prominent plateaulike region as seen in the thin curve. There is no indication in the appearance of the thick curve in Fig. 2 that the cluster passes through a potential energy extremum at any stage of its trajectory.

It must be emphasized that the method described above does not determine the transition state geometry precisely. Two factors are responsible for this. First, since we are attempting to describe the dynamics of the system with a finite number of Fourier coefficients, we are not determining the true dynamics of the system. Second, the system is required to pass through a transition state (or an extremum) only in the limit of an infinitely long transit time. To locate the transition state precisely, we pick a configuration in the transition-state (plateau) region of the trajectory and mini- 
mize the sum of the squares of the gradients of the potential. Usually, the potential energy of the initially chosen configuration is within $0.05 \epsilon$ of the actual potential energy of the transition state. The transition state is confirmed by diagonalizing the Hessian matrix and verifying that there is one negative eigenvalue corresponding to the reaction coordinatc, along with six zero eigenvalues corresponding to rotations and translations and a remaining set of positive eigenvalues corresponding to the bound normal modes.

\section{RESULTS}

\section{A. Seven-atom cluster}

We have used the double-ended classical trajectory method to locate transition states between potential energy minima of the seven-atom Lennard-Jones cluster. The sevenatom cluster has four minima, two of which have been shown in Fig. 1. Previous studies by Davis et al. ${ }^{6}$ found eight transition states connecting these four minima with the use of the slowest-slide ${ }^{8}$ and Cerjan-Miller ${ }^{5}$ methods. In testing our method, we have located all previously reported transition states among the isomers of the 7-atom Lennard-Jones cluster. All trajectory searches were carried out with three expansion coefficients for each Cartesian coordinate. Since the presence of a plateaulike region in the plot of the potential energy along the trajectory can only suggcst an cxtremum on the potential energy surface and not necessarily a transition state, we did not know a priori whether a trajectory was in fact a transition-state trajectory. Once a possible transitionstate trajectory was identified, the geometry of the cluster in the plateaulike region of the trajectory was optimized by minimizing the sum of the squares of the gradients. Once this sum was sufficiently small, the eigenvalues of the Hessian matrix were evaluated to determine whether the extremum was a transition state or a higher order saddle point.

We have also located a transition state not reported previously. The structure of this transition state is shown in Fig. 3 . This transition state that connects the tricapped tetrahedron with itself [see Fig. 1(c) of Ref. 6], has a potential energy of $-14.57 \epsilon$ and is similar to the transition state [show'n in Fig. 2(h) of Ref. 6] previously found that connects the bicapped trigonal bipyramid with itself [see Fig. 1(d) of Ref. 6]. In both cases, the transition state occurs via the edge-bridging mechanism. ${ }^{9}$

In addition to the transition states, we have also found a second-order saddle point in the potential energy surface between the lowest-energy isomer, the pentagonal bipyramid, and the tricapped tetrahedron. What makes this particular saddle point, shown in Fig. 4, interesting is the fact that its potential energy of $-14.75 \epsilon$ is lower than that of two previously reported transition states shown in Figs. $2(\mathrm{~g})$ and 2(h) of Ref. 6 and the transition state shown in Fig. 3 of this paper. This new saddle point can be expected to contribute to the low-energy dynamics of the 7-atom cluster on an equal footing with energetically similar transition states.

\section{B. Eight-atom cluster}

A number of transition states among the four lowestenergy isomers of the eight-particle Lennard-Jones cluster

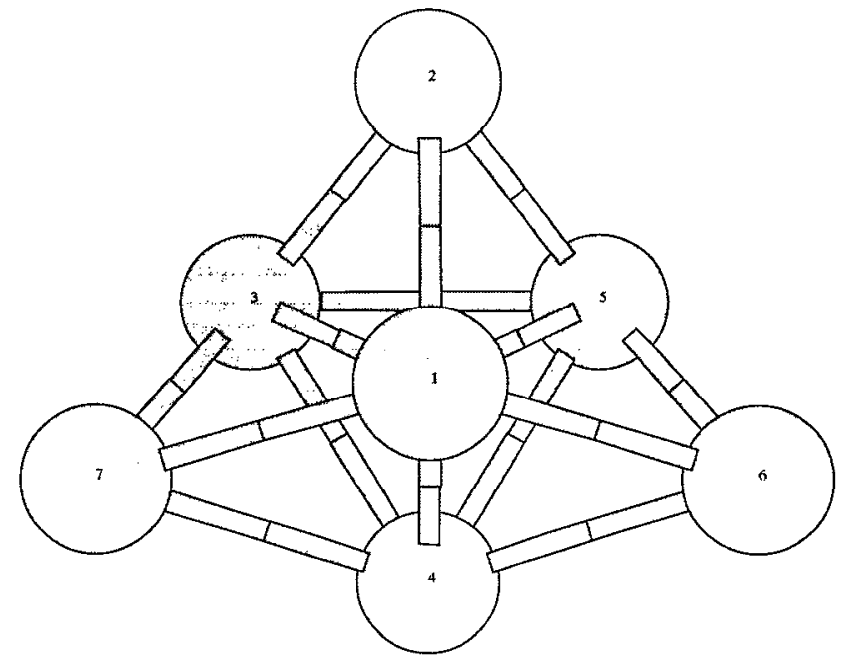

FIG. 4. Geometry at the second-order saddle point between the pentagonal bipyramid and the capped octahedron, $V=-14.75 \epsilon$.

have been found by Wales. ${ }^{7}$ We found two previously unreported transition states between the capped pentagonal bipyramid and the bicapped octahedron. The structures for these minima are shown in Figs. 3(a) and 3(c) of Ref. 7, respectively, and their potential energies are $-19.83 \epsilon$ and $-19.19 \epsilon$. The transition states, shown in Figs. 5(a) and 5(b), both have lower energies $[-18.71 \epsilon$ and $-18.68 \epsilon$, respeclively] than the previously reported transition state between these two minima [ $-18.42 \epsilon$, shown in Fig. 4(d) of Ref. 7].

The search for the transition states of the 8-atom cluster also revealed a limitation of our method. We were unable to locate a transition-state trajectory between the dodecahedron and the bicapped octahedron, shown Figs. 4(b) and 4(c) of Ref. 7 , respectively. The lowest-energy trajectory that we found connecting these two structures did not yield a potential energy along the trajectory characteristic of a transitionstate trajectory. This curve, shown in Fig. 6, does not exhibit the characteristics of the lower (thin) curve in Fig. 2. Further investigation of geometries along the trajectory corresponding to the plot in Fig. 6 revealed that the configuration of the cluster at a time corresponding to the small shoulderlike feature in Fig. 6 (at $u \sim .25$ ) was close to the previously reported transition state, shown in Fig. 4(b) of Ref. 7. The transitionstate trajectory should, therefore, have its maximum potential energy at that configuration.

In an effort to understand the reason behind the inability of our method to locate the transition state between the bicapped octahedron and the dodecahedron, we conducted a search for trajectories connecting each of the two potential energy minima with the transition state. Ideally, the potential energy along the lowest-energy trajectory from a potential energy minimum to a transition state should increase monotonically. This was the case for the trajectory originating at the bicapped octahedron. A trajectory with monotonically increasing potential energy starting at the dodecahedron, however, could not be located. The lowest-energy trajectory that was found between the dodecahedron and the transition state 

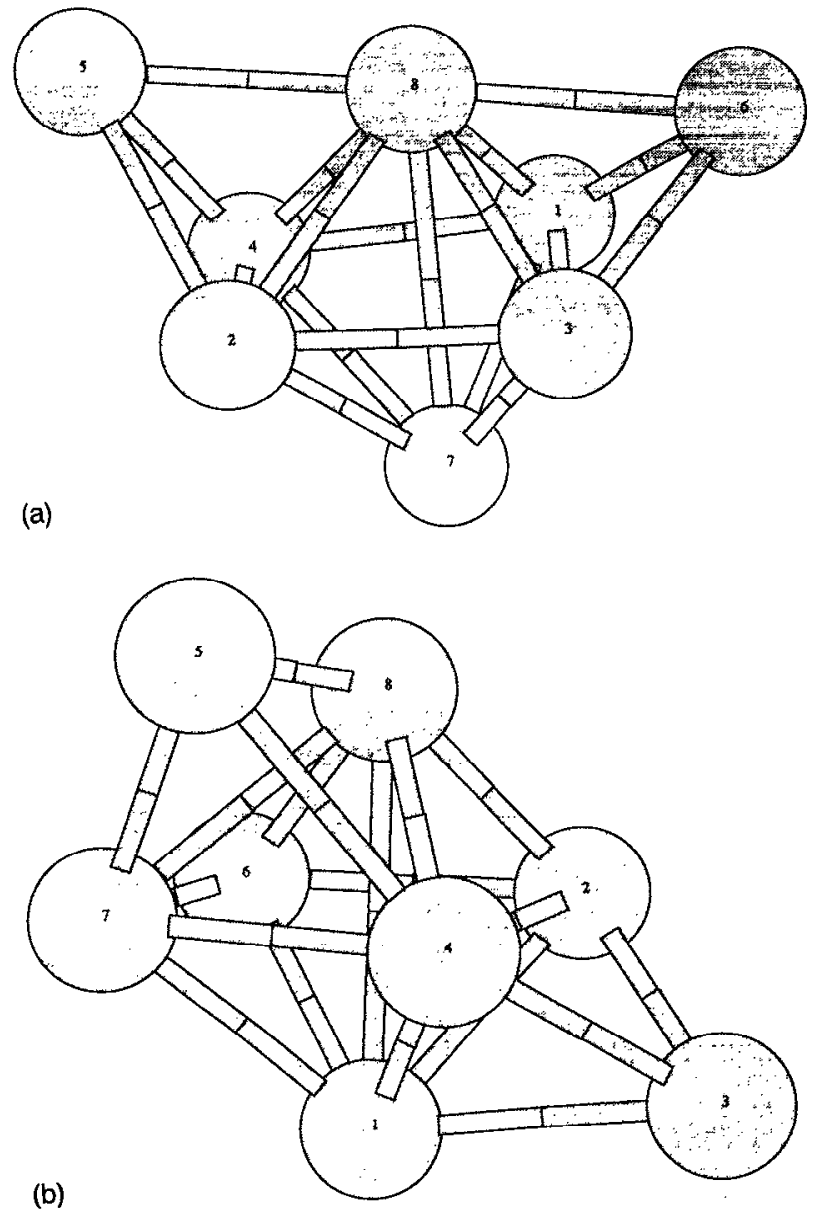

FIG. 5. Transition states for the 8-atom cluster between the capped pentago-

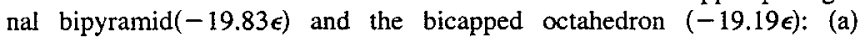
$-18.71 \epsilon,(b)-18.68 \epsilon$.

passed through configurations with potential energies above that of the transition state.

To study this case in more detail we ran molecular dynamics trajectories (using standard coordinate-momentum boundary conditions) starting at configurations slightly displaced from the transition-state geometry along the reaction coordinate. The atoms in the clusters were given no initial momenta. When the configuration was displaced toward the bicapped octahedron, the cluster immediately found the corresponding potential energy minimum. On the other hand, when the starting geometry was displaced toward the dodecahedron, the lowest potential energy reached by the cluster before a subsequent increase was $0.4 \epsilon$ above the corresponding potential energy minimum. The results of the standard molecular dynamics trajectories together with the lack of a transition-state trajectory between these two minima imply that a dynamical transition-state trajectory connecting these two minima does not exit. More specifically, the transition state is not dynamically accessible from the minimum corresponding to the dodecahedron with a trajectory whose total energy does not exceed the potential energy at the transition state. This result implies that a careful search for transition states along the lowest energy trajectory is warranted even when the lowest energy trajectory does not have the characteristics of a transition-state trajectory.

\section{CONCLUDING REMARKS}

We have presented a novel approach for locating transition states in a multi-dimensional potential. The doubleended classical trajectory approach has been adapted to search efficiently for trajectories that take the system from one potential energy minimum to another through or close to an extremum on the potential energy surface. With this technique, we have been able to locate a previously unreported transition state for the 7-atom Lennard-Jones cluster and two previously unreported transition states for the 8-atom cluster.

We have also tested our approach on the 13-atom cluster and were able to locate several of the transition states reported in Ref. 7. We did not conduct an extensive search for the transition states of the 13-particle cluster because we were mainly interested in determining the applicability of our method to larger clusters. The typical times for determining the set of expansion coefficients for a trajectory ranged from under half a minute for the 7-atom cluster to about 2 minutes for a 13-atom cluster on a Silicon Graphics Indigo workstation. The computing time scaled as the square of the number of atoms and, therefore, significantly larger systems are certainly accessible with this method.

The appeal of our method is the ability to use physical intuition in choosing the relative orientations and numbering of atoms in a pair of potential energy minima between which a transition state is being sought. This way one can force the cluster to undergo a specific rearrangement and determine whether a transition state exists for such a path. We have also learned that a successful application of this method relies on the existence of a dynamical transition-state trajectory. As it

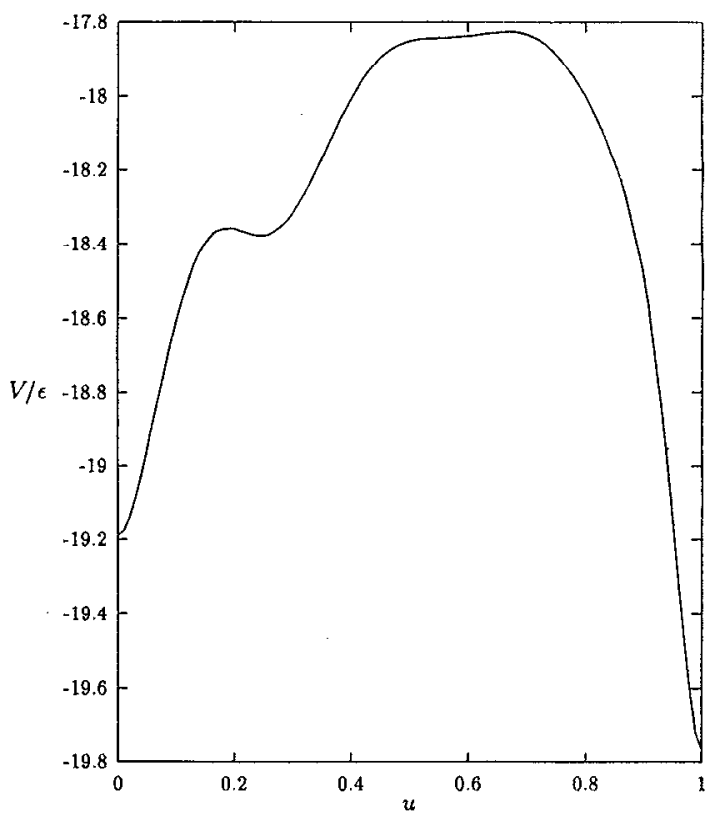

FIG. 6. Potential energy along the lowest-entergy trajectory for the rearrangement between the dodecahedron and the bicapped octahedron minima of the 8-atom cluster. 
turned out, a transition state for the 8-atom cluster was not dynamically accessible from one of the minima without passing through configurations with potential energies above that of the transition state. This implies the need for a careful search of the lowest energy trajectory to seek any hidden transition states.

Note added in proof. After this work was accepted for publication, we became aware of the work of Tsai and Jordan, ${ }^{12}$ who located the transition states for the seven-and eight-atom Lennard-Jones clusters we stated had not previously been reported. We thank Dr. D. J. Wales for making this reference known to us.

\section{ACKNOWLEDGMENTS}

Acknowledgment is made to the Donors of the Petroleum Research Fund of the American Chemical Society for support of this work. This work was also supported by NSF Grant No. CHE-9203498. Figures 1, 3, 4 and 5 were drawn using XMol, version 1.3.1, Minnesota Supercomputer Center, Inc., Minneapolis, MN, 1993.

${ }^{1}$ J. D. Doll, T. L. Beck, and D. L. Freeman, Int. J. Quantum Chem., Quantum Chem. Symp. 23, 73 (1989).

${ }^{2}$ A. E. Cho, J. D. Doll, and D. L. Freeman, Chem. Phys. Lett (in press).

${ }^{3}$ R. E. Gillian and K. R. Wilson, J. Chem. Phys. 97, 5713 (1992).

${ }^{4}$ L. R. Pratt, J. Chem. Phys. 85, 5045 (1986).

${ }^{5}$ C. J. Cerjan and W. H. Miller, J. Chem. Phys. 75, 2800 (1981).

${ }^{6}$ H. L. Davis, D. J. Wales, and R. S. Berry, J. Chem. Phys. 92, 4308 (1990).

${ }^{7}$ D. J. Wales, J. Chem. Phys. 91, 7002 (1989).

${ }^{8}$ R. S. Berry, H. L. Davis, and T. L. Beck, Chem. Phys. Lett. 147, 13 (1988).

${ }^{9}$ B. F. G. Johnson, J. Chem. Soc., Chem. Commun. 1986, 27 (1986).

${ }^{10}$ W. N. Lipscomb, Science 153, 373 (1966).

${ }^{11}$ W. H. Press, S. A. Teukolski, W. T. Vetterling, and B. P. Flannery, Numerical Recipes (Cambridge University, Cambridge, 1986), Chap. 10.

${ }^{12}$ C. J. Tsai and K. D. Jordan, J. Phys. Chem. 97, 11227 (1993). 
The Journal of Chemical Physics is copyrighted by the American Institute of Physics (AIP). Redistribution of journal material is subject to the AIP online journal license and/or AIP copyright. For more information, see http:/ojps.aip.org/jcpo/jcpcr/jsp Copyright of Journal of Chemical Physics is the property of American Institute of Physics and its content may not be copied or emailed to multiple sites or posted to a listserv without the copyright holder's express written permission. However, users may print, download, or email articles for individual use. 
The Journal of Chemical Physics is copyrighted by the American Institute of Physics (AIP). Redistribution of journal material is subject to the AIP online journal license and/or AIP copyright. For more information, see http://ojps.aip.org/jcpo/jcper/jsp 